\title{
ON THE STRUCTURE OF THE SET OF SUBSEQUENTIAL LIMIT POINTS OF SUCCESSIVE APPROXIMATIONS
}

\author{
BY J. B. DIAZ AND F. T. METCALF ${ }^{1}$
}

Communicated February 8, 1967

1. Introduction. Let $A: S \rightarrow S$ be continuous, where $S$ is a nonempty metric space. Much current research is concerned with the existence of fixed points of $A$. Given $x \in S$, it is natural to ask if the (Picard) sequence of iterates $\left\{A^{m}(x)\right\}_{m=1}^{\infty}$ converges to a fixed point of $A$. In certain circumstances, answers to questions of this general type have been given by Edelstein [1], [2] and Browder and Petryshyn [3], [4]. It appears to be of interest to consider first the structure of the set of subsequential limit points of the sequence of iterates $\left\{A^{m}(x)\right\}_{m=1}^{\infty}$, with the ultimate end in view of ascertaining when this sequence actually converges. This is the general approach followed in this announcement.

2. Main results. It is quite popular to consider a function $A$ which is a contraction over its entire domain of definition. The following theorem makes use of a contractive hypothesis, but only with respect to the set of fixed points of $A$ (see hypothesis (ii) below).

In the sequel, $F(A)$ will denote the set of fixed points of $A$, that is, $F(A)=\{x \in S \mid A(x)=x\}$. Also, $d(x, F(A))$ will denote the distance between the point $x$ and the set $F(A)$, that is, $d(x, F(A))$ $=\inf _{p \in F(A)} d(x, p)$. Further, for $x \in S, \mathfrak{L}(x)$ will denote the set of subsequential limit points of the sequence of iterates $\left\{A^{m}(x)\right\}_{m=1}^{\infty}$. As usual, a subset $K$ of $S$ will be said to be compact if every sequence of points from $K$ contains a subsequence which converges to a point in $S$.

Theorem 1. Suppose

(i) $F(A)$ is nonempty and compact;

(ii) for each $x \in S$, with $x \notin F(A)$, one has

$$
d(A(x), F(A))<d(x, F(A)) .
$$

Then, for $x \in S$, the set $\mathfrak{L}(x)$ is a closed and connected subset of $F(A)$. Either $\mathfrak{L}(x)$ is empty, or it contains exactly one point, or it contains

1 This research was supported in part by the Air Force Office of Scientific Research under Grants AFOSR 1122-66 and 1122-67. 
uncountably many points. In case $\mathfrak{L}(x)$ is just one point, then $\lim _{m \rightarrow \infty} A^{m}(x)$ exists and belongs to $F(A)$. In case $\mathfrak{L}(x)$ is uncountable, then it is contained in the boundary of $F(A)$.

OUTLINE OF PROOF. If $\mathscr{L}(x)$ is empty, then there is nothing to prove. Suppose, therefore, that $\mathscr{L}(x)$ is nonempty. It can then be shown in succession that: (1) $\mathscr{L}(x)$ is a subset of $F(A) ;(2) \mathscr{L}(x)$ is closed; (3) $\mathscr{L}(x)$ is connected; (4) $\mathscr{L}(x)$ is a single point or is uncountable; (5) $\mathscr{L}(x)$ consists of just one point implies that $\lim _{m \rightarrow \infty} A^{m}(x)$ exists and belongs to $F(A) ;(6) \mathfrak{L}(x)$, if uncountable, lies in the boundary of $F(A)$.

Upon making additional hypotheses, several corollaries of this theorem are obtained, of which only two will be mentioned: (1) If $S$ is compact, then it immediately follows that $\mathscr{L}(x)$ is nonempty; (2) If $F(A)$ is countable, then either every subsequence of $\left\{A^{m}(x)\right\}_{m=1}^{\infty}$ converges (necessarily to the same point of $F(A)$ ) or every subsequence of $\left\{A^{m}(x)\right\}_{m=1}^{\infty}$ diverges.

A related theorem is obtained by weakening hypothesis (i) and strengthening hypothesis (ii). The net result is a strengthening of the conclusion of Theorem 1.

Theorem 2. Suppose

(i) $F(A)$ is nonempty;

(ii) for each $x \in S$, with $x \notin F(A)$, and each $p \in F(A)$, one has

$$
d(A(x), p)<d(x, p)
$$

Then, for $x \in S$, either every subsequence of $\left\{A^{m}(x)\right\}_{m=1}^{\infty}$ converges (necessarily to the same point of $F(A))$ or every subsequence of $\left\{A^{m}(x)\right\}_{m=1}^{\infty}$ diverges. That is, either $\mathfrak{L}(x)$ contains exactly one point or $\mathcal{L}(x)$ is empty.

A variant of Theorem 1 is obtained by allowing equality in hypothesis (ii) of Theorem 1 and employing the notion of "asymptotic regularity" introduced by Browder and Petryshyn [4, p. 572, Definition 1] in dealing with Banach spaces.

THEOREM 3. Suppose

(i) $F(A)$ is nonempty and compact;

(ii) for each $x \in S$, one has $d(A(x), F(A)) \leqq d(x, F(A))$;

(iii) for each $x \in S$, one has $\lim _{m \rightarrow \infty} d\left(A^{m+1}(x), A^{m}(x)\right)=0$. Then, for $x \in S$, the set $\mathfrak{L}(x)$ is a closed and connected subset of $F(A)$. Either $\mathfrak{L}(x)$ is empty, or it contains exactly one point, or it contains uncountably many points. In case $\mathfrak{L}(x)$ is just one point, then $\lim _{m \rightarrow \infty} A^{m}(x)$ exists and 
belongs to $F(A)$. In case $\mathfrak{L}(x)$ is uncountable, then it is contained in the boundary of $F(A)$.

A variant of Theorem 2 , which is related to Theorem 2 in the same way that Theorem 3 is related to Theorem 1, can also be obtained, but will not be stated explicitly here.

3. Applications. Theorem 2 can be applied to obtain a result of Edelstein [2] which extends an earlier result of Krasnosel'skir [5].

As another application of Theorem 2, one can obtain the following theorem, which is closely related to a result of Fridman [6] for Fredholm integral equations of the first kind.

THEOREM 4. Let $T$ be a continuous function on a nonempty Hilbert space $H$, and with values also in $H$. Let $y \in H$. Suppose

(i) there exists a $p \in H$ such that $T p=y$;

(ii) there exists a number $\lambda>0$ such that, for each $x \in H$, with $T x \neq y$, and each $p \in H$, with $T p=y$, one has

$$
\frac{1}{2} \lambda\|T x-T p\|^{2}<\operatorname{Re}(T x-T p, x-p) .
$$

Let $x_{0} \in H$. Then the sequence $\left\{x_{m}\right\}_{m=0}^{\infty}$, where

$$
x_{m+1}=x_{m}+\lambda\left(y-T x_{m}\right), \quad m=0,1, \cdots,
$$

either contains no (strongly) convergent subsequence, or else $\left\{x_{m}\right\}_{m=0}^{\infty}$ is (strongly) convergent and $T\left(\lim _{m \rightarrow \infty} x_{m}\right)=y$.

4. Concluding remarks. This investigation had its origin in the stimulating series of lectures delivered by Professor F. Tricomi at the Institute for Fluid Dynamics and Applied Mathematics, University of Maryland, in April of 1966. In the course of his lectures Professor Tricomi drew our attention to his first published paper [7], which is concerned with the iteration of a real-valued function.

It is expected that complete proofs of the announced results will appear in full elsewhere.

\section{REFERENCES}

1. M. Edelstein, On fixed and periodic points under contractive mappings, J. London Math. Soc. 37 (1962), 74-79.

2. - A remark on a theorem of Krasnoselski, Amer. Math. Monthly 73 (1966), 509-510.

3. F. E. Browder and W. V. Petryshyn, The solution by iteration of linear functional equations in Banach spaces, Bull. Amer. Math. Soc. 72 (1966), 566-570.

4. - The solution by iteration of nonlinear functional equations in Banach spaces, Bull. Amer. Math. Soc. 72 (1966), 571-575. 
5. M. A. Krasnosel'skiY, Two remarks on the method of successive approximations, Uspehi Mat. Nauk 10 (1955), No. 1, 123-127.

6. V. M. Fridman, The method of successive approximations for Fredholm integral equations of the first kind, Uspehi Mat. Nauk 11 (1956), No. 1, 233-234.

7. F. Tricomi, Un teorema sulla convergenza delle successioni formate delle successive iterate di una funzione di una variabile reale, Giorn. Mat. Battaglini 54 (1916), 1-9.

University OF CALIFornia, RIVERside. 